\title{
In Silico Investigation of Spontaneous Calcium Release on Premature Ventricular Contractions in Human Ventricles
}

\author{
Jieyun Bai ${ }^{1}$, Kuanquan Wang ${ }^{1}$, Gongning Luo ${ }^{1}$, Henggui Zhang ${ }^{1,2}$ \\ ${ }^{1}$ School of Computer Science and Technology, Harbin Institute Technology, Harbin, China \\ ${ }^{2}$ School of Physics and Astronomy, University of Manchester, Manchester, UK
}

\begin{abstract}
Aim: Sarcoplasmic reticulum (SR) calcium overload increases spontaneous calcium release, causing delayed afterdepolarizations (DADs) that promote premature ventricular contractions (PVCs). In this study, we modified a detailed human ventricular model to investigate quantitatively how SR calcium features at the subcellular scale influence cellular DADs, facilitating the onset of PVCs at the tissue level.

Methods: Based on the 4-state Shannon model, calcium-induced-calcium release flow into the dyadic cleft via the SR ryanodine receptor (RyR2) in Ten Tusscher and Panfilov (TP06) model was modified. SR calcium release was varied by changing $S R$ calcium content for simulating beta-adrenergic stimulation. The cellular DAD amplitude required to depolarize the cell to threshold and trigger an action potential (AP) was quantified. A one-dimensional cable model, which contained a central area of contiguous myocytes susceptible to DADs, was constructed to investigate requirements for DADs to overcome the source-sink mismatch and trigger PVCs.

Results and Conclusion: Depending on the content of SR calcium, SR calcium release in cardiac cell resulted in DADs. When the amplitude of a DAD is above a certain threshold (20.7 $\mathrm{mV}$, from $-86.2 \mathrm{mV}$ to $-65.5 \mathrm{mV}$ ), a suprathreshold $D A D$ in single cell can trigger an $A P$, which can cause a PVC in cardiac tissue. However, the subthreshold DADs didn't produce APs but formed a conduction block region. The number of contiguous susceptible myocytes required for a suprathreshold DAD to trigger a propagating AP is 76 in a cardiac cable with 100 cells, corresponding to $11.4 \mathrm{~mm}$. The number was significantly decreased by increased SR calcium load, reduced gap junction and fibrosis. In conclusion, $S R$ calcium overload caused by electrical remodeling in combination with slow conduction induced by structural remodeling decreased the number significantly but still require synchronization mechanisms for DADs to overcome the source-sink mismatch to trigger PVCs.
\end{abstract}

\section{Introduction}

Arrhythmias are a major cause of sudden cardiac death. Three-dimensional mapping indicates that most of ventricular tachycardia are initiated by the focal mechanism. This often connected to special excitations of cardiac cells (abnormal ventricular automaticity or triggered activity). The triggered activity includes early afterdepolarizations (EADs) occurring in the plateau phase of the action potential (AP) and delayed afterdepolarizations (DADs) occurring after complete repolarization.

DADs, the focus in the present study, are generally thought to be initiated by spontaneous calcium release from the sarcoplasmic reticulum (SR) and a calciumactivated transient, depolarizing inward current as a result of SR calcium overload. Previous electrophysiological studies in isolated ventricular myocytes suggested that spontaneous calcium release from the SR can evoke triggered arrhythmias. Although DADs are generally accepted to be calcium-dependent, the relationship between SR calcium release and DAD amplitude has not been measured, partly because the underlying calcium transients are hard to control. In addition, DADs occurring at the cellular level cannot be extrapolated directly to focal arrhythmias occurring at the tissue level, because the questions as to how the source of excitation from these cellular DADs overcomes the sink of the surrounding neighbored cells to be depolarized to produce focal arrhythmias in the heart remains incompletely understood.

In the present study, we used beta-adrenergic signaling-induced SR calcium overload to simulate DADs with different SR calcium concentration $\left(\left[\mathrm{Ca}^{2+}\right]_{\mathrm{SR}}\right)$ in a much more controlled manner and measured the resulting depolarization. These DADs can be initiated at various SR calcium loads, allowing us to measure the $\left[\mathrm{Ca}^{2+}\right]_{\mathrm{SR}}$ dependence of DADs and triggered APs over a broad range of $\left[\mathrm{Ca}^{2+}\right]_{\mathrm{SR}}$. To investigate how DADs are synchronized to overcome the source-sink mismatch and produce focal arrhythmia in the tissue level, we 
constructed a mathematical model to determine whether spontaneous calcium release produces spatiotemporal synchronization of DADs and to examine cell-cell uncoupling on the induction of focal arrhythmia.

The goals of the present study are measure, (1) the quantitative relationship between the amount of SR calcium release and the amplitude of DADs, (2) the amount of SR calcium release required to reach threshold for an AP, (3) the specific contributions of SR calcium release and cell-cell uncoupling that cause focal arrhythmias, and (4) the effect of source-sink interactions on the induction of focal arrhythmia. The results provide the quantitative data on the basis of DADs and focal arrhythmia in human ventricles.

\section{Method and material}

\subsection{Action potential model}

A one-dimensional (1D) human ventricular model was developed based on the ten Tusscher and Panfilov (TP06) ventricular cell model. Some parameters were modified to simulate SR calcium overload.

Since according to ten Tusscher et al., $I_{Y \text {, base is basal }}$ flow without calcium/calmodulin-dependent protein kinase II (CaMKII) activation, then:

$$
I_{i}=G_{i} \times m_{i} \times n_{i} \times\left(V-V_{r e v, i}\right)
$$

where $I_{i}$ is the current of the ion channel $i, G_{i}$ is the maximal conductance of $i$ channel, $m_{i}$ and $n_{i}$ are gates of the $i$ channel, $V$ is the cellular membrane potential and $V_{r e v, i}$ is the reversal potential for $i$ channel.

Since according to O'Hara et al., $f_{\text {CaMKII }}$ is the CaMKII factor, then:

$$
f_{\text {CaMKII }}=1+\frac{I F_{\text {CaMKII }}}{1+K_{\text {mCaMKII }} / \text { CaMKII }}
$$

where $K_{m \text { CaMKII }}$ denotes half-saturation concentration of CaMKII and CaMKII act is fraction of activated CaMKII. Details of CaMKII kinetics may be found in Hund et al. For $f_{\text {CaMKII }}$ calculation, mean $I F_{\text {CaMKII }}$ was 0.25 for L-type calcium channel $\left(I_{C a L}\right), 0.05$ for SR calcium release $\left(I_{\text {rel }}\right)$, 0.45 for SR calcium ATPase2a $\left(I_{u p}\right), 0.08$ for transient out current $\left(I_{t o}\right)$, and 0.2 for late sodium flow $\left(I_{N a L}\right)$. in:

Incorporating $I_{i}$ and $f_{\text {СамKII }}$ into total flow $\left(I_{I}\right)$, results

$$
I_{I}=f_{\text {CaMKII }} \times I_{i}
$$

that takes into account both CaMKII effects on the corres ponding ion flows.

\section{2. $\quad 1 D$ tissue model}

To investigate requirements for DADs at the cellular level to trigger a PVC in tissue, we employ a model of $1 \mathrm{D}$ epicardial strand of $15 \mathrm{~mm}$ (100 myocytes with length of
$0.15 \mathrm{~mm}$ ). In the model, the center subdomain including a variable number of contiguous cells tuned to exhibit DADs and the surrounding regions representing normal tissue. The maximum contents of $\left[\mathrm{Ca}^{2+}\right]_{\mathrm{SR}}$ associated with these DADs were measured to evaluate the inducibility of AP. The threshold for DADs to trigger an AP was computed as the amplitude of DADs required to active the sodium channel. The minimum number of susceptible cells (subthreshold or suprathreshold DADs) required to develop a PVC in the cable was calculated. Effects of gap junction on the minimum number required to initiate a PVC were performed to evaluate the sink-source relationship of cardiac tissue. Change of gap junction was implemented by decreasing diffusion coefficient from 100\% (\#1) to 80\% (\#2), 60\% (\#3), 40\% (\#4) and 20\% (\#5).

We simulated electrical activation and propagation in a $1 \mathrm{D}$ virtual tissue, governed by the following reactiondiffusion equation:

$$
C_{m} \partial V / \partial t=D \nabla^{2} V-I_{\text {ion }}
$$

where $V(\mathrm{mV})$ is the transmembrane potential, $C_{m}(\mathrm{pF})$ is the membrane capacitance, $I_{\text {ion }}(\mathrm{pA})$ is the sum of ionic currents(see Formula (1)) and $t(\mathrm{~ms})$ is time, and $D$ is the electronic diffusion modelling gap junction coupling. More detailed parameters and equations were documented in the TP06 model.

\subsection{Numerical methods}

For computational purposes, the tissue was discretized in $100 \mu \mathrm{m} \times 100 \mu \mathrm{m}$ patches and a time-step of $2 \mu \mathrm{s}$ was used. We solved Eq (4) and the TP06 equations using an explicit Euler method, with both a lookup table of the voltage dependent parameters in the TP06 model and the operator splitting technique. "No-flux" boundary conditions were applied. Simulations were carried out on a 64 G memory with Intel core i703930K 64-bit CPU system. Efficient parallelization was implemented using GPU acceleration.

\section{Results}

\subsection{Dependence of depolarization on SR calcium release}

SR calcium load available for release was varied by changing pacing frequency. Figure 1 shows the last steady-state AP at frequencies of 1 to $3 \mathrm{~Hz}$. As expected, there is a progressive increase in twitch $\left[\mathrm{Ca}^{2+}\right]_{\mathrm{SR}}$ with increasing frequency. After the last stimulated AP, DADs were induced at $2 \mathrm{~Hz}$ and $3 \mathrm{~Hz}$. With increasing frequency, the content of $\left[\mathrm{Ca}^{2+}\right]_{\mathrm{SR}}$ increased, consistent with the expected increase in SR calcium load. For $1-\mathrm{Hz}$ stimulation, a normal AP was observed (Figure 1A). The 
subthreshold DAD was induced as $\left[\mathrm{Ca}^{2+}\right]_{\mathrm{sR}}$ rose at $2 \mathrm{~Hz}$ (Figure 1B). At $4 \mathrm{~Hz}$, depolarization was sufficient to trigger a regenerative AP (Figure $1 \mathrm{C}$ ).

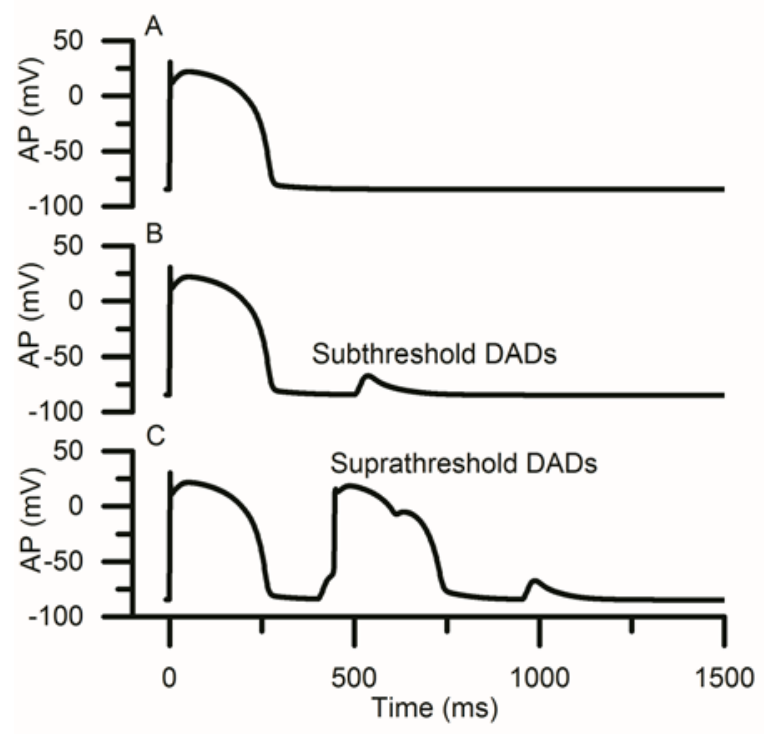

Figure 1. Cellular simulation of action potentials (APs) with 1-, 2-, and 3-Hz stimulation. A: Normal AP at $1 \mathrm{~Hz}$; B: Subthreshold DAD was induced high-frequency (2Hz); C: Suprathreshold DAD was triggered by SR calcium overload at $3 \mathrm{~Hz}$.

\subsection{Requirements for DADs to trigger PVCs in a 1D homogeneous tissue}

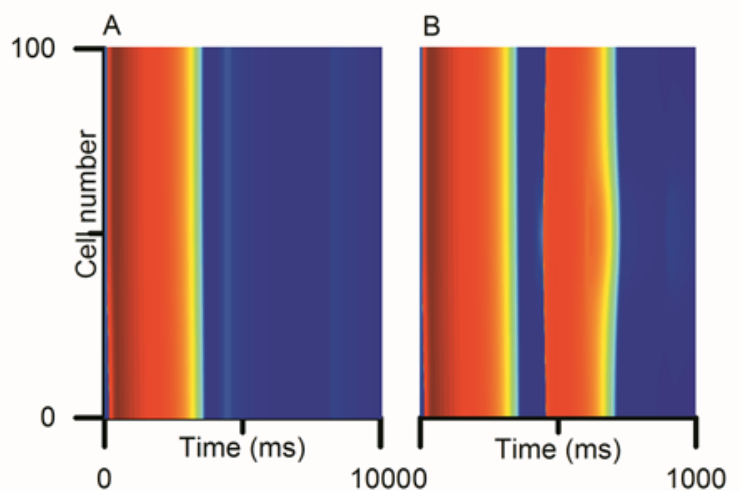

Figure 2. Requirements for DADs to trigger excitation waves in well-couple $1 \mathrm{D}$ cable. A: The subthreshold DAD did not trigger an excitation wave; $B$ : The suprathreshold DAD successfully propagated into the surrounding tissue.

To investigate how local epicardium with DADs overcomes electronic source-sink mismatches to trigger PVCs, we constructed a 1D epicardial tissue model in which the central region contained cells susceptible to DADs. The outer surrounding regions contained normal cells. The number of susceptible myocytes in the central region was then progressively increased until DADs triggered APs that propagated successfully into the surrounding normal tissue. For the subthreshold DADs, although cells in the cable were susceptible to subthreshold DADs, the DAD failed to trigger a PVC (Figure 2A). Same as for subthreshold DADs, enough suprathreshold DAD cells successfully overcome the sink-source mismatch to produce an excitation wave (Figure 2B).

\subsection{Effects of SR calcium overload and cell-cell uncoupling on the inducibility of PVC: source-sink relationship}

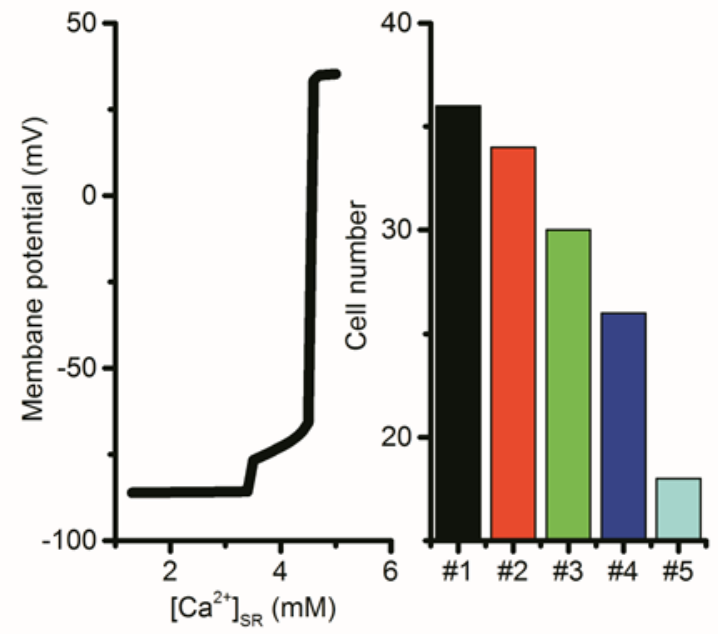

Figure 3. Effect of SR calcium content and gap junction uncoupling on the inducibility of PVCs. A: Depolarized potential increased with SR calcium load; B: The cell number required for DADs to trigger PVCs significantly decreased with gap junction uncoupling. \#1: 100\% gap junction; \#2: 80\% gap junction; \#3: 60\% gap junction; \#4: 40\% gap junction; \#5: 20\% gap junction.

Subcellular SR function analysis has shown that DAD amplitude is generally to be $\left[\mathrm{Ca}^{2+}\right]_{\mathrm{SR}}$-dependent. Augmented SR calcium load increased spontaneous SR calcium release, promoting membrane depolarization toward the threshold to trigger an AP, the precursor of PVC (Figure 3). The minimum of peak $\left[\mathrm{Ca}^{2+}\right]_{\mathrm{sR}}$ contributed to the threshold DAD amplitude $(20.7 \mathrm{mV}$, from $-86.2 \mathrm{mV}$ to $-65.5 \mathrm{mV}$ ) required to depolarize the cell to trigger an AP was $4.45 \mathrm{mM}$ (Figure 3A). For the suprathreshold DAD case, the length required to trigger a propagating excitation wave was $11.4 \mathrm{~mm}$, corresponding to 76 myocytes. However, if the total SR calcium was increased to augment the DAD amplitude further above the AP threshold, the required number of cells decreased significantly.

To investigate the effect of gap junction uncoupling on the development of PVCs, we performed analogous 
simulations by using $100 \%, 80 \%, 60 \%, 40 \%$ and $20 \%$ of the diffusion coefficient, which reduced conduction velocity $(\mathrm{CV})$ from $74.2 \mathrm{~cm} / \mathrm{s}$ to $65.8 \mathrm{~cm} / \mathrm{s}, 56.3 \mathrm{~cm} / \mathrm{s}, 45$ $\mathrm{cm} / \mathrm{s}$ and $30.1 \mathrm{~cm} / \mathrm{s}$, respectively. When peak SR calcium was $5 \mathrm{mM}$, the size of the central region was significantly reduced. In detail, the cell number of the susceptible region required to trigger a PVC reduced from 36 to 34, 30, 26 and 18 (Figure 3B). This effect can be attributable to decreased excitability and conduction which led to less 'sink' of tissue, corresponding to less 'source' required to trigger a PVC and smaller region of susceptible cells compared to the normal condition.

\section{Discussions and conclusion}

In the present study, we characterized quantitatively the relationship between $\left[\mathrm{Ca}^{2+}\right]_{\mathrm{SR}}$ and membrane depolarization in a setting that mimics DADs, and important precursor of triggered arrhythmias. We confirmed that spatiotemporal synchronization of spontaneous calcium release produced focal activity in tissue level. Source-sink interactions were found to be critically important in the generation of calcium-mediated focal arrhythmias.

\section{Acknowledgements}

This work is supported by the National Natural Science Foundation of China (NSFC) under Grant No. 61571165 and No. 61572152.

\section{References}

[1]. Turakhia MP. Sudden cardiac death and implantable cardioverter-defibrillators. American family physician 2010;82:1357-66.

[2]. Gaztañaga L, Marchlinski FE, Betensky BP. Mechanisms of cardiac arrhythmias. Revista Española de Cardiología
(English Edition) 2012;65:174-85.

[3]. Shiferaw Y, Aistrup GL, Wasserstrom JA. Intracellular $\mathrm{Ca} 2+$ waves, afterdepolarizations, and triggered arrhythmias. Cardiovascular research 2012;95:265-8.

[4]. Song Z, Ko CY, Nivala M, Weiss JN, Qu Z. CalciumVoltage Coupling in the Genesis of Early and Delayed Afterdepolarizations in Cardiac Myocytes. Biophysical journal 2015;108:1908-21.

[5]. Weiss JN, Garfinkel A, Karagueuzian HS, Chen P-S, Qu Z. Early afterdepolarizations and cardiac arrhythmias. Heart Rhythm 2010;7:1891-9.

[6]. Huffaker R, Lamp ST, Weiss JN, Kogan B. Intracellular calcium cycling, early afterdepolarizations, and reentry in simulated long QT syndrome. Heart Rhythm 2004;1:4418.

[7]. Huffaker RB, Weiss JN, Kogan B. Effects of early afterdepolarizations on reentry in cardiac tissue: a simulation study. American Journal of Physiology-Heart and Circulatory Physiology 2007;292:H3089-H102.

[8]. Mahajan A, Shiferaw Y, Sato D, et al. A rabbit ventricular action potential model replicating cardiac dynamics at rapid heart rates. Biophysical journal 2008;94:392-410.

[9]. de Lange E, Xie Y, Qu Z. Synchronization of early afterdepolarizations and arrhythmogenesis in heterogeneous cardiac tissue models. Biophysical journal 2012;103:365-73.

[10]. Vandersickel N, Kazbanov IV, Nuitermans A, Weise LD, Pandit R, Panfilov AV. A study of early afterdepolarizations in a model for human ventricular tissue. PloS one 2014;9:e84595.

[11]. ten Tusscher KH, Panfilov AV. Alternans and spiral breakup in a human ventricular tissue model. American Journal of Physiology-Heart and Circulatory Physiology 2006;291:H1088-H100.

Address for correspondence.

Kuanquan Wang

Mailbox 332, Harbin Institute of Technology Harbin, China wangkq@hit.edu.cn 